\title{
Survey of the training and use of echocardiography and lung ultrasound in Australasian intensive care units
}

\author{
Yang Yang ${ }^{1,2^{*}}$ (D), Colin Royse $e^{1,4}$, Alistair Royse ${ }^{1,4}$, Kacey Williams ${ }^{3}$ and David Canty ${ }^{1,4}$
}

\author{
Abbreviations: FCU, Focused Cardiac Ultrasound; ICU, Intensive Care Unit; LU, Lung Ultrasound; \\ TOE, Transoesophageal Echocardiography; TTE, Transthoracic Echocardiography
}

\section{Introduction}

Transthoracic echocardiography (TTE) and focused cardiac ultrasound (FCU) are now considered essential skills and a requirement of training for physicians working in the intensive care unit (ICU) [1]. TTE is a feasible and safer alternative to transoesophageal echocardiography (TOE), even after cardiac surgery [2]. Acquiring competency in TTE during an already over-full curriculum is a challenge. Furthermore, lung ultrasound (LU) is becoming established for bedside diagnosis of acute respiratory pathology [3]. The current level of practice and training in TTE, TOE and LU in ICU is not yet reported. We surveyed the 114 ICUs accredited for ICU training in Australasia to determine the current prevalence of practice and training in TTE, TOE, and LU and to identify perceived obstacles in practice and training. After ethics approval, a web-based survey of 14 Multiple Choice Questions was submitted to the Directors of ICU accredited for training.

\section{Findings}

Out of 114 ICUs, 69 (61\%) completed the survey, including $23(33 \%)$ that admitted cardiothoracic surgical patients and $44(67 \%)$ that did not. The proportion of ICUs performing TTE (94\%) and LU was high (83 \%) but the use of TOE was low (33\%). The proportion of ICU consultants performing TTE, LU, and TOE was lower $(42 \%, 51 \%$, and $10 \%)$, respectively. The level of

\footnotetext{
* Correspondence: Y.Yang@wh.org.au

${ }^{1}$ Ultrasound Education Group, Department of Surgery, The University of

Melbourne, Melbourne, Victoria, Australia

${ }^{2}$ Intensive Care Unit, Western Health, Eastern Health, Epworth Private

Hospital, Melbourne, Victoria, Australia

Full list of author information is available at the end of the article
}

expertise (diagnostic versus focused) was highest in TTE (32\%) compared with TOE (22\%) and LU (12\%).

The proportions of intensivists untrained in TTE and LU was $41 \%$ and $30 \%$, respectively. Perceived barriers included lack of organized training (38\%) and time for training $(25 \%)$. Other barriers included a perceived lack of need for training (18\%), insufficient equipment (14\%), and resistance from other ultrasound providers $(4 \%)$. The most commonly reported training programs were tertiary courses, such as provided by the Australasian Society of Ultrasound in Medicine (68 \%) and University of Melbourne (59\%), rather than board examinations or hands-on workshops.

We conclude that although TTE and LU are used frequently in Australasian teaching ICUs, many ICU physicians are yet to be trained due to lack of ICU training programs and time for training. Although tertiary courses are popular and provide training to diagnostic level, they are lengthy and depend on trainers and patient caseload and are not, therefore, scalable. An attractive alternative is to begin training in medical school and to train more physicians in basic ultrasound with shorter, more efficient, and hands-on courses utilizing the internet and ultrasound simulators [1], advancing to a diagnostic level only if required.

\section{Additional file}

Additional file 1: Qualtrics Survey Software. (PDF $138 \mathrm{~kb}$ )

\section{Acknowledgements}

The authors would like to thank the clinicians and administrative staffs who responded to the survey. 


\section{Funding}

No funding.

\section{Availability of data and materials}

Supporting data are available as Additional file 1.

\section{Authors' contributions}

DC: study design, supervision of data collection, analysis, and manuscript. YY: study design, supervision of data collection, analysis, and manuscript. KW: contribution to study design, performance of data collection. CR: contribution to study design, analysis, and manuscript. AR: contribution to analysis and manuscript. All authors read and approved the final manuscript.

\section{Competing interests}

Colin Royse and Alistair Royse are employees of University of Melbourne and are involved in the development and delivery of ultrasound courses such as Graduate Certificate, Diploma and Masters of Clinical Ultrasound and iHeartScan ${ }^{T M}$ and are co-directors of Heartweb, which conducts the SCA ON-CUE courses. David Canty is an employee of University of Melbourne and is involved in the development and delivery of ultrasound courses such as Graduate Certificate, Diploma and Masters of Clinical Ultrasound and iHeartScan ${ }^{\text {TM }}$. Yang Yang is an Honorary Lecturer of the University of Melbourne and is involved in ultrasound training.

\section{Consent for publication}

The authors consent the survey for publication.

\section{Ethical approval and consent to participate}

Approved by Melbourne Health Human Research Ethics Committee as a quality assurance project (QA2015082) for conducting and publication of the result of the publication. Informed consent was obtained from participants responding to the survey (Additional file 1).

\section{Author details}

${ }^{1}$ Ultrasound Education Group, Department of Surgery, The University of Melbourne, Melbourne, Victoria, Australia. ${ }^{2}$ Intensive Care Unit, Western Health, Eastern Health, Epworth Private Hospital, Melbourne, Victoria, Australia. ${ }^{3}$ Department of Anaesthesia, St. Michael's Hospital, Toronto, Ontario, Canada. ${ }^{4}$ Department of Anaesthesia, The Royal Melbourne Hospital, Melbourne, Victoria, Australia.

\section{Published online: 24 October 2016}

\section{References}

1. Mayo PH. Training in critical care echocardiography. Ann Intensive Care. 2011;1:36.

2. Costachescu T, Denault A, Guimond JG, Couture P, Carignan S, Sheridan P, et al. The haemodynamically unstable patient in the intensive care unit: haemodynamic vs. transesophageal echocardiographic monitoring. Crit Care Med. 2002;30:1214-23.

3. Alsaddique A, Royse AG, Royse CF, Mobeirek A, El Shaer F, AlBackr H, et al. Repeated monitoring with transthoracic echocardiography and lung ultrasound after cardiac surgery: feasibility and impact on diagnosis. J Cardiothorac Vasc Anesth. 2016;30(2):406-12. doi:10.1053/j.jvca.2015.08.033. Epub 2015 Aug 29. 